\title{
Young nephron progenitors might extend the lifespan of old progenitors
}

Exposure of old nephron progenitor cells (postnatal day 0 ) to a young progenitor cell niche (12.5 days post-coitum) extends the capacity of the older cells for selfrenewal, according to new research from Raphael Kopan and colleagues. These findings suggest that younger nephron progenitors can reset the age of older cells to enable additional nephron production.

The researchers used heterochronic transplantation of differentially labelled young and old progenitor cells into an unlabelled young niche, and single-cell mRNA analysis, to study the behaviour of individual cells relative to each other. "If old progenitor cells had an immutable internal clock they would all depart from the niche when their classmates did, but if they were true stem cells that were forced out of the niche by external cues they would remain in a 1:1 ratio of young to old cells for as long as the niche permitted," explains Kopan. "Instead, we observed an in-between result-some of the old progenitor cells preferentially exited the cap mesenchyme niche, but $\sim 30 \%$ of these cells remained and continued to contribute to nephron production for up to 1 week after engraftment." Interestingly, the older cells that extended their progenitor potential tended to be isolated from each other, and were often surrounded by younger cells.

"We now aim to determine which molecular pathways underlie this observed progenitor cell juxtacrine signalling mechanism," says Kopan. "Such data could eventually contribute to strategies to increase nephron numbers in at-risk populations, such as those born prematurely or those with low birth weights."

Jessica K. Edwards

Original article Chen, S. et al. Intrinsic age-dependent changes and cell-cell contacts regulate nephron progenitor lifespan. Dev. Cell 25, 49-62 (2015) 